\title{
Current experimental status
}

\author{
Aurelio Juste ${ }^{* \dagger}$ \\ Fermi National Accelerator Laboratory \\ E-mail: justeefnal.gov
}

Ten years after its discovery at the Tevatron collider, we still know little about the top quark. Its large mass suggests it may play a key role in the mechanism of Electroweak Symmetry Breaking (EWSB), or open a window of sensitivity to new physics related to EWSB and preferentially coupled to it. To determine whether this is the case, precision measurements of top quark properties are necessary. The high statistics samples being collected by the Tevatron experiments during Run II start to incisively probe the top quark sector. This report summarizes the experimental status of the top quark, focusing in particular on the recent measurements from the Tevatron.

International Workshop on Top Quark Physics

January 12-15, 2006

Coimbra, Portugal

* Speaker.

${ }^{\dagger}$ The author would like to thank the organizers for their invitation and a stimulating and enjoyable conference. 


\section{Introduction}

The top quark vas discovered in 1995 by the CDF and DØ collaborations[1] during Run I of the Fermilab Tevatron collider. Like any discovery, this one caused a big excitement, although it did not really come as a surprise: the top quark existence was already required by self-consistency of the Standard Model (SM). One of the most striking properties of the top quark is its large mass, comparable to the Electroweak Symmetry Breaking (EWSB) scale. Therefore, the top quark might be instrumental in helping resolve one of the most urgent problems in High Energy Physics: identifying the mechanism of EWSB and mass generation. In fact, the top quark may either play a key role in EWSB, or serve as a window to new physics related to EWSB and which, because of its large mass, might be preferentially coupled to it. Ten years after its discovery, we still know little about the top quark: existing indirect constraints on top quark properties from low-energy data, or the statistics-limited direct measurements at Tevatron Run I, are relatively poor and leave plenty of room for new physics. Precision measurements of top quark properties are crucial in order to unveil its true nature. Currently, the Tevatron collider is the world's only source of top quarks and a comprehensive program of measurements is well underway.

\section{The Tevatron Accelerator}

The Tevatron is a proton-antiproton $(p \bar{p})$ collider operating at a center of mass energy of 1.96 $\mathrm{TeV}$. With respect to Run I, the center of mass energy has been slightly increased (from $1.8 \mathrm{TeV}$ ) and the inter-bunch crossing reduced to $396 \mathrm{~ns}$ (from $3.6 \mu \mathrm{s}$ ). The latter and many other upgrades to Fermilab's accelerator complex have been made to significantly increase the luminosity. Since the beginning of Run II in March 2001, the Tevatron has delivered an integrated luminosity of $\sim 1.3$ $\mathrm{fb}^{-1}$, and is currently operating at instantaneous luminosities $\mathscr{L}>1.5 \times 10^{32} \mathrm{~cm}^{-2} \mathrm{~s}^{-1}$. The goal is to collect $\sim 4.1-8.2 \mathrm{fb}^{-1}$ by the end of 2009 . This represents a $\times 40-80$ increase with respect to the Run I data set, which will allow the Tevatron experiments to make the transition from the discovery phase to a phase of precision measurements of top quark properties.

\section{Top Quark Production and Decay}

At the Tevatron, the dominant production mechanism for top quarks is in pairs $(t \bar{t})$, mediated by the strong interaction, with a predicted cross section of $\sigma_{t \bar{t}}=6.77 \pm 0.42 \mathrm{pb}$ for $m_{t}=175 \mathrm{GeV}$.[2] Within the SM, top quarks can also be produced singly via the electroweak (EW) interaction, with $\sim 40 \%$ of the $t \bar{t}$ production rate. However, single top quark production has not been discovered yet. While the production rate of top quarks at the Tevatron is relatively high, $\sim 2 t \bar{t}$ events/hour at $\mathscr{L}=1 \times 10^{32} \mathrm{~cm}^{-2} \mathrm{~s}^{-1}$, this signal must be filtered out from the $\sim 7 \mathrm{M}$ inelastic $p \bar{p}$ collisions per second. This underscores the importance of highly efficient and selective triggers.

Since $m_{t}>M_{W}$, the top quark in the SM almost always decays to an on-shell $W$ boson and a $b$ quark. The dominance of the $t \rightarrow W b$ decay mode results from the fact that, assuming a 3generation and unitary CKM matrix, $\left|V_{t s}\right|,\left|V_{t d}\right|<<\left|V_{t b}\right| \simeq 1$.[3] The large mass of the top quark also results in a large decay width, $\Gamma_{t} \simeq 1.4 \mathrm{GeV}$ for $m_{t}=175 \mathrm{GeV}$, which leads to a phenomenology radically different from that of lighter quarks. Because $\Gamma_{t}>>\Lambda_{Q C D}$, the top quark decays before 
top-flavored hadrons or $t \bar{t}$-quarkonium bound-states have time to form. As a result, the top quark provides a unique laboratory, both experimentally and theoretically, to study the interactions of a bare quark. Thus, the final state signature of top quark events is completely determined by the $W$ boson decay modes. In the case of $t \bar{t}$ decay, the three main channels considered experimentally are referred to as dilepton, lepton plus jets and all-hadronic, depending on whether both, only one or none of the $W$ bosons decayed leptonically. The dilepton channel has the smallest branching ratio, $\sim 5 \%$, and is characterized by two charged leptons ( $e$ or $\mu$ ), large transverse missing energy $\left(B_{T}\right)$ because of the two undetected neutrinos, and $\geq 2$ jets (additional jets may result from initial or final state radiation). The lepton plus jets channel has a branching ratio of $\sim 30 \%$ and is characterized by one charged lepton ( $e$ or $\mu$ ), large $E_{T}$ and $\geq 4$ jets. The largest branching ratio, $\sim 46 \%$, corresponds to the all-hadronic channel, characterized by $\geq 6$ jets. In all instances, two of the jets result from the hadronization of the $b$ quarks and are referred to as $b$-jets. As it can be appreciated, the detection of top quark events requires a multipurpose detector with excellent lepton, jet and $b$ identification capabilities, as well as hermetic calorimetry with good energy resolution.

\section{The CDF and DØ Detectors}

The CDF and DØ detectors from Run I already satisfied many of the requirements for a successful top physics program. Nevertheless, they underwent significant upgrades in Run II in order to further improve acceptance and $b$ identification capabilities, as well as to cope with the higher luminosities expected.[4] CDF has retained its central calorimeter and part of the muon system, while it has replaced the central tracking system (drift chamber and silicon tracker). A new plug calorimeter and additional muon coverage extend lepton identification in the forward region. DØ has completely replaced the tracking system, installing a fiber tracker and silicon tracker, both immersed in a $2 \mathrm{~T}$ superconducting solenoid. $\mathrm{D} \emptyset$ has also improved the muon system and installed new preshower detectors. Both CDF and D $\varnothing$ have upgraded their DAQ and trigger systems to accommodate the shorter inter-bunch time.

\section{Top Quark Pair Production Cross Section}

The precise measurement of $\sigma_{t \bar{t}}$ is a key element of the top physics program. It provides a test of perturbative QCD and a sensitive probe for new physics effects affecting both top quark production and decay. Especially for the latter, the comparison of measurements in as many channels as possible is crucial. Also, by virtue of the detailed understanding required in terms of object identification and backgrounds, cross section analyses constitute the building blocks of any other top quark properties measurements.

The measurements performed by CDF and D $\varnothing$ in Run I at $\sqrt{s}=1.8 \mathrm{TeV}[5]$ were found to be in good agreement with the SM prediction, but limited in precision to $\sim 25 \%$ as a result of the low available statistics. In Run II, the large expected increase in statistics will yield measurements a priori only limited by systematic uncertainties. These include jet energy calibration, signal/background modeling, luminosity determination, etc. However, it is also expected that such large data samples will allow to control/reduce many of these systematic uncertainties. The goal in Run II is to achieve a per-experiment uncertainty of $\Delta \sigma_{t \bar{t}} / \sigma_{t \bar{t}} \leq 10 \%$ for $\simeq 2 \mathrm{fb}^{-1}$. 

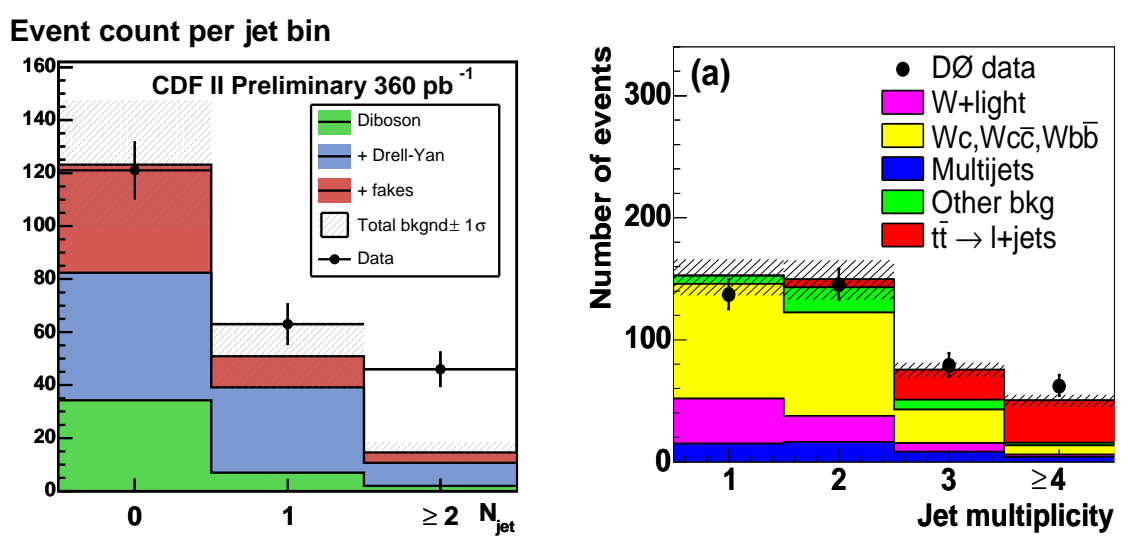

Figure 1: Left: Jet multiplicity distribution for $t \bar{t}$ candidate events selected in the $\ell+$ track channel (CDF). Right: Jet multiplicity distribution for $t \bar{t}$ candidate events selected in the lepton plus jets channel, requiring $\geq 1$ jet to be $b$-tagged by a secondary vertex algorithm (DØ).

\subsection{Dilepton Final States}

Typical event selections require the presence of two high $p_{T}$ isolated leptons $(e, \mu, \tau$ or isolated track), large $E_{T}$ and $\geq 2$ high $p_{T}$ central jets. Physics backgrounds to this channel include processes with real leptons and $\not_{T}$ in the final state such as $Z / \gamma^{*} \rightarrow \tau^{+} \tau^{-}(\tau \rightarrow e, \mu)$ and diboson production $(W W, W Z, Z Z)$. The dominant instrumental backgrounds result from $Z / \gamma^{*} \rightarrow e^{+} e^{-}, \mu^{+} \mu^{-}$, with large $E_{T}$ arising from detector resolution effects, and processes where one or more jets fake the isolated lepton signature ( $W+j e t s$ or QCD multijets). Additional kinematic or topological cuts are usually applied to further reduce backgrounds, such as e.g on $H_{T}$ (sum of $p_{T}$ of jets in the event), exploiting the fact that jets from $t \bar{t}$ are energetic, whereas for backgrounds they typically arise from initial state radiation and have softer $p_{T}$ spectra. CDF and DØ have developed different analysis techniques to exploit the potential of the sample. The standard dilepton analysis $(\ell \ell)$, where two well identified leptons ( $e$ or $\mu$ ) and $\geq 2$ jets are required, has high purity $(S / B \geq 3)$ but reduced statistics because of the stringent requirements made. In order to improve the signal acceptance, the so-called lepton+track analysis $(\ell+$ track) demands only one well identified lepton and an isolated track, and $\geq 2$ jets (see Fig. 1(left)). This analysis has increased acceptance for taus, in particular 1-prong hadronic decays.

\subsection{Lepton Plus Jets Final States}

Typical event selections require one high $p_{T}$ isolated lepton ( $e$ or $\mu$ ), large $E_{T}$ and $\geq 3$ high $p_{T}$ central jets. The dominant background is $W+$ jets, followed by QCD multijets with one of the jets faking a lepton. After selection the signal constitutes $\sim 10 \%$ of the sample. Further signal-tobackground discrimination can be achieved by exploiting the fact that all $t \bar{t}$ events contain two $b$ quarks in the final state whereas only a few percent of background events do. CDF and D $\varnothing$ have developed $b$-tagging techniques able to achieve high efficiency and background rejection: lifetime tagging and soft-lepton tagging. Lifetime tagging techniques rely upon $B$ mesons being massive and long-lived, traveling $\sim 3 \mathrm{~mm}$ before decaying with high track multiplicity. The high resolution 
Table 1: Summary of the best $\sigma_{t \bar{t}}$ measurements at Tevatron Run II.

\begin{tabular}{|c|c|c|c|c|}
\hline Channel & Method & $\sigma_{t \bar{t}}(\mathrm{pb})$ & $L\left(\mathrm{pb}^{-1}\right)$ & Experiment \\
\hline Dilepton & $\ell+$ track & $9.9 \pm 2.1$ (stat.) \pm 1.4 (syst.) & 360 & CDF \\
& $\ell \ell$ & $8.6_{-2.0}^{+2.3}$ (stat. $)_{-1.0}^{+1.2}$ (syst.) & 370 & $\mathrm{D} \emptyset$ \\
\hline Lepton plus Jets & SVT & $8.2 \pm 0.9$ (stat.) \pm 0.9 (syst.) & 363 & DØ \\
& SMT & $5.2_{-1.9}^{+2.9}$ (stat.) $)_{-1.0}^{+1.3}$ (syst.) & 193 & CDF[6] \\
& Kinematic & $6.3 \pm 0.8$ (stat.) \pm 1.0 (syst.) & 347 & CDF \\
\hline All-Hadronic & SVT & $8.0 \pm 1.7$ (stat.) $)_{-2.2}^{+3.3}$ (syst.) & 311 & CDF \\
\hline \hline Prelim. Combination & N/A & $7.1 \pm 0.6$ (stat.) \pm 0.8 (syst.) & 350 & CDF \\
\hline
\end{tabular}

vertex detector allows to directly reconstruct secondary vertices significantly displaced from the event primary vertex (secondary vertex tagging, or SVT) or identify displaced tracks with large impact parameter significance. Soft-lepton tagging is based on the identification within a jet of a soft electron or muon resulting from a semileptonic $B$ decay. Only soft-muon tagging (SMT) has been used so far, although soft-electron tagging is under development and should soon become available. The performance of the current algorithms can be quantified by comparing the event tagging probability for $t \bar{t}$ and the dominant $W+j e t s$ background. For instance, for events with $\geq 4$ jets: $P_{\geq 1-t a g}(t \bar{t}) \simeq 60 \%(16 \%)$ whereas $P_{\geq 1-t a g}(W+$ jets $) \simeq 4 \%$, using SVT(SMT). These analyses are typically pure counting experiments and are performed as a function of jet multiplicity in the event (see Fig. 1(right)). Events with 3 or $\geq 4$ jets are expected to be enriched in $t \bar{t}$ signal, whereas events with only 1 or 2 jets are expected to be dominated by background. The former are used to estimate $\sigma_{t \bar{t}}$, and the latter to verify the background normalization procedure.

$\mathrm{CDF}$ and $\mathrm{D} \emptyset$ have also developed analyses exploiting the kinematic and topological characteristics of $t \bar{t}$ events to discriminate against backgrounds: leptons and jets are more energetic and central and the events have a more spherical topology. The statistical sensitivity is maximized by combining several discriminant variables into a multivariate analysis (e.g. using neural networks), where $\sigma_{t \bar{t}}$ is extracted from a fit to the discriminant distribution using templates from MC. Some of the dominant systematic uncertainties (e.g. jet energy calibration) can be reduced by making more inclusive selections (e.g. $\geq 3$ jets instead of $\geq 4$ jets). The combination of both approaches to improve statistical and systematic uncertainties have for the first time yielded measurements competitive with those using $b$-tagging (see Table 1).

\subsection{All-Hadronic Final State}

Despite its spectacular signature with $\geq 6$ high $p_{T}$ jets, the all-hadronic channel is extremely challenging because of the overwhelming QCD multijets background $(S / B \sim 1 / 2500)$. Nevertheless, CDF and DØ successfully performed measurements of $\sigma_{t \bar{t}}$ and the top quark mass in this channel in Run I. Current measurements by CDF and DØ focus on the $b$-tagged sample and make use of kinematic and topological information to further increase the signal-to-background ratio. CDF applies cuts on a set of four discriminant variables, whereas DØ builds an array of neural networks. In both cases, background is directly predicted from data. 


\subsection{Summary}

Table 1 presents a summary of the best measurements in Run II in each of the different decay channels. So far, the different measurements are in agreement with each other and with the SM prediction. As precision continues to increase, the detailed comparison among channels will become sensitive to new physics effects. The single most precise measurement (lepton plus jets/SVT) has already reached $\Delta \sigma_{t \bar{t}} / \sigma_{t \bar{t}} \sim 16 \%$ and starts becoming systematics-limited. There is much work underway to further reduce systematic uncertainties as well as to combine measurements.

\section{Top Quark Mass}

The top quark mass $\left(m_{t}\right)$ is a fundamental parameter of the SM, not predicted by the theory, and should be measured to the highest possible accuracy. It plays an important role in precision EW analyses, where some observables such as $M_{W}$ receive loop corrections $\propto m_{t}^{2}$. This fact was originally exploited to predict the value of $m_{t}$ before the top quark discovery, which was ultimately found to be in good agreement with the experimental measurements and constituted a significant success of the SM. After the top quark discovery, the precise measurements of $m_{t}$ and $M_{W}$ can be used to constrain the value of the mass of the long-sought Higgs boson $\left(M_{H}\right)$, since some of the EW precision observables also receive quantum corrections $\propto \log \left(M_{H}\right)$. An uncertainty of $\Delta m_{t} \leq 2.0$ $\mathrm{GeV}$ would indirectly determine $M_{H}$ to $\sim 30 \%$ of its value.

Achieving such high precision is not an easy task, but the experience gained in Run I and the much improved detectors and novel ideas in Run II provide a number of handles that seem to make this goal reachable. In Run I, the dominant systematic uncertainty on $m_{t}$ was due to the jet energy scale calibration. The reason is that the top quark mass measurement requires a complicated correction procedure (accounting for detector, jet algorithm and physics effects) to provide a precise mapping between reconstructed jets and the original partons. To determine and/or validate the jet energy calibration procedure, data samples corresponding to di-jet, $\gamma+j e t s$ and $Z+j e t s$ production were extensively used. In addition to the above, the large $t \bar{t}$ samples in Run II allow for an in situ calibration of light jets making use of the $W$ mass determination in $W \rightarrow j j$ from top quark decays, a measurement whose precision is in principle expected to scale as $1 / \sqrt{N}$. Also, dedicated triggers requiring displaced tracks will allow to directly observe $Z \rightarrow b \bar{b}$, which can be used to verify the energy calibration for $b$ jets. Additional important requirements for a precise $m_{t}$ measurement are an accurate detector modeling and state-of-the-art theoretical knowledge (gluon radiation, $b$ fragmentation, etc). The golden channel for a precise measurement is provided by the lepton plus jets final state, by virtue of its large branching ratio and moderate backgrounds, as well as the presence of only one neutrino, which leads to over-constrained kinematics. Powerful $b$-tagging algorithms are being used to reduce both physics and combinatorial backgrounds, and sophisticated mass extraction techniques are being developed, resulting in improvements in statistical as well as systematic uncertainties. An overview of the main analysis methods is given below.

The so-called "Template Methods", traditionally used in Run I, start by constructing an eventby-event variable sensitive to $m_{t}$, e.g. the reconstructed top quark mass from a constrained kinematic fit in the lepton plus jets channel. The top quark mass is extracted by comparing data to templates on that particular variable built from MC for different values of $m_{t}$. Recent developments in this approach by CDF (see Fig. 2(left)) have lead to the single most precise measurement 


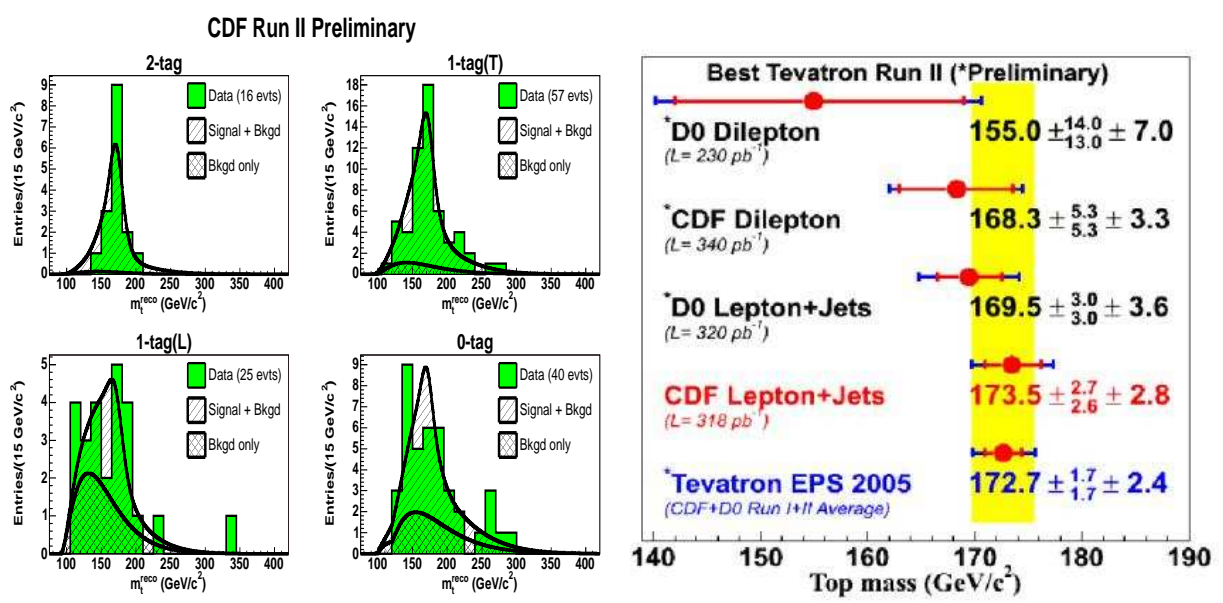

Figure 2: Left: Reconstructed $m_{t}$ distribution from a constrained kinematical fi $\mathrm{t}$ in the lepton plus jets channel (CDF). The distribution is shown separately for the different subsamples defi ned based on the $b$-tag multiplicity. Right: Summary of the best $m_{t}$ measurements at Tevatron Run II.

to date:[7] $m_{t}=173.5_{-3.6}^{+3.7}$ (stat. $\left.+\mathrm{JES}\right) \pm 1.3$ (syst.) GeV. The statistical uncertainty is minimized by separately performing the analysis in four subsamples with different $b$-tag multiplicity, thus each with a different background content and sensitivity to $m_{t}$. The dominant systematic uncertainty, jet energy calibration (JES), is reduced by using the in situ $W$ mass determination from $W \rightarrow j j$ in a simultaneous fit of $m_{t}$ and a jet energy calibration factor. The latter is also constrained to a $\sim 3 \%$ precise external measurement in control samples. The remaining systematic uncertainties, amounting to $\Delta m_{t}=1.3 \mathrm{GeV}$, include contributions such as background shape, $b$-fragmentation, gluon radiation, etc, many of which are expected to be further reduced with larger data samples.

The so-called "Dynamic Methods" have as main objective making an optimal used of the statistical information in the sample. They are based on the calculation of the per-event probability density as function of $m_{t}$, taking into account resolution effects (better measured events contribute more) and summing over all permutations of jets as well as neutrino solutions. These methods typically include a complete or partial matrix element evaluation for the signal and dominant background processes. The so-called Matrix Element Method was pioneered by D $\varnothing$ and applied to the lepton plus jets Run I sample[8], leading to the single most precise measurement in Run I. In Run II, both CDF and DØ have applied this method to the lepton plus jets sample, yielding results competitive with the template method discussed above. CDF has also applied this method to the dilepton sample,[9] yielding $m_{t}=165.2 \pm 6.1$ (stat.) \pm 3.4 (syst.) $\mathrm{GeV}$.

Fig. 2(right) summarizes the best Run II measurements from CDF and DØ in the different channels. As it can be appreciated, some of the Run II individual measurements are already reaching a precision comparable or better than the Run I world average.[10] The new preliminary combination of CDF and DØ Run I and Run II measurements in all channels yields[11]: $m_{t}=172.7 \pm 2.9$ $\mathrm{GeV}, \chi^{2} / d o f=6.5 / 7$. The resulting constraints on the SM Higgs boson mass are: $M_{H}=91_{-32}^{+45}$ $\mathrm{GeV}$ or $M_{H}<186 \mathrm{GeV}$ at $95 \%$ C.L.. Based on the current experience with Run II measurements, it is expected that an uncertainty of $\Delta m_{t} \leq 1.5 \mathrm{GeV}$ can be achieved at the Tevatron with $2 \mathrm{fb}^{-1}$. 


\section{Top Quark Charge}

The top quark charge, one of the most fundamental quantities characterizing a particle, has not been directly measured yet. In particular, there is no guarantee that the selected $t \bar{t}$ candidate events correspond to pair production of resonances with $Q= \pm 2 e / 3$. One possibility[12] is that the "discovered top quark" is actually an exotic quark with $Q=-4 e / 3$, and thus decays to $W^{-} b$ instead of $W^{+} b$. Based on $365 \mathrm{pb}^{-1}$ of data selected in the lepton plus $\geq 4$ jets channel and requiring $\geq 2 b$-tagged jets, $\mathrm{D} \emptyset$ has made use of a jet charge algorithm and constrained kinematic fitting to exclude the hypothesis of $Q=-4 e / 3$ at $94 \%$ C.L..

\section{Top Quark Couplings to the $W$ boson}

If the top quark is indeed playing a special role in the EWSB mechanism, it may have non-SM interactions to the weak gauge bosons. At the Tevatron, only the interaction of the top quark to the $W$ boson can be sensitively probed. Within the SM, the charged-current interactions of the top quark are of the type $\mathrm{V}-\mathrm{A}$ and completely dominated by the $t W b$ vertex by virtue of the fact that $\left|V_{t b}\right| \simeq 1$. In fact, the $t W b$ vertex defines most of the top quark phenomenology: it determines the rate of single top quark production and completely saturates the top quark decay rate. It is also responsible for the large top quark width, that makes it decay before hadronizing, thus efficiently transmitting its spin to the final state. The angular distributions of the top quark decay products also depend on the structure of the $t W b$ vertex.

\subsection{Single Top Quark Production}

Within the SM, the main production mechanisms for single top quarks at the Tevatron involve the exchange of a time-like $W$ boson (s-channel), $\sigma_{s}=0.88 \pm 0.07 \mathrm{pb}$, or a space-like $W$ boson (tchannel), $\sigma_{t}=1.98 \pm 0.21 \mathrm{pb}$.[13] Despite the relatively large expected rate, single top production has not been discovered yet. The experimental signature is almost identical to the lepton plus jets channel in $t \bar{t}$ : one high $p_{T}$ isolated lepton, large $\not_{T}$ and jets, but with lower jet multiplicity (typically 2 jets) in the final state, which dramatically increases the $W+$ jets background. In addition, $t \bar{t}$ production becomes a significant background with a very similar topology (e.g. if one lepton in the dilepton channel is not reconstructed).

Once it is discovered, the precise determination of the single top production cross section will probe, not only the Lorentz structure, but also the magnitude of the $t W b$ vertex, thus providing the only direct measurement of $\left|V_{t b}\right|$. The sensitivity to anomalous top quark interactions is enhanced by virtue of the fact that top quarks are produced with a high degree of polarization. In addition, the s- and t-channels are differently sensitive to new physics effects,[14] so the independent measurement of $\sigma_{s}$ and $\sigma_{t}$ would allow to discriminate among new physics models should any deviations from the SM be observed.

In Run II the search for single top quark production continues[15] with ever increasing data samples, improved detector performance, and increasingly more sophisticated analyses. The generic analysis starts by selecting $b$-tagged lepton plus $\geq 2$ jets candidate events. CDF considers one discriminant variable per channel (e.g. $Q(\ell) \times \eta$ (untagged jet) for the t-channel search) whereas $\mathrm{D} \emptyset$ performs multivariate analyses. The upper limit on $\sigma$ is estimated exploiting the shape of the 

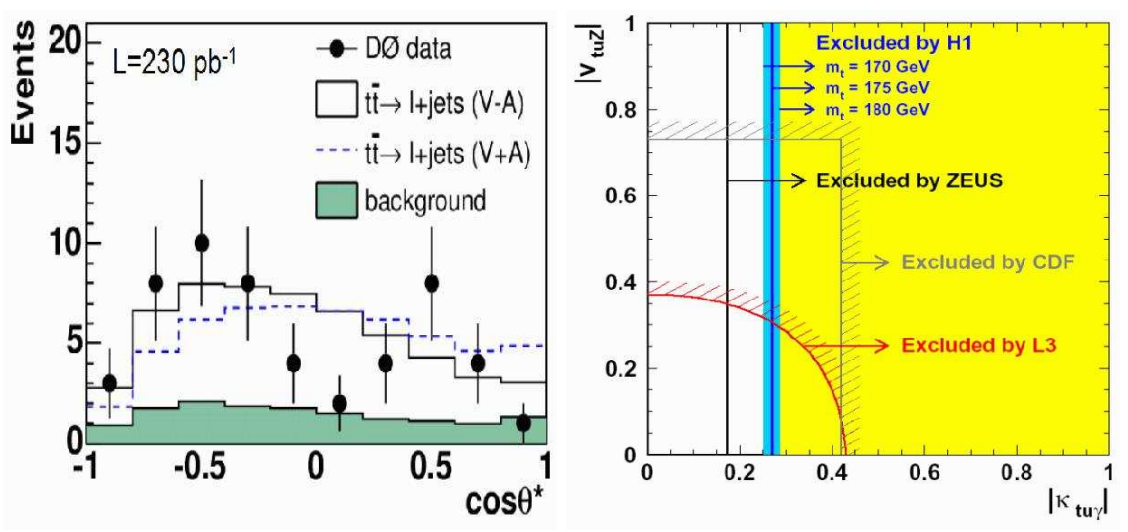

Figure 3: Left: Lepton helicity angle distribution in the the $b$-tagged lepton plus $\geq 4$ jets sample (DØ). Right: Exclusion limits at the 95\% C.L. on the anomalous $t u Z$ and $t u \gamma$ couplings obtained at the Tevatron, LEP (only L3 experiment shown) and HERA.

discriminant variable and using a Bayesian approach. Using $\sim 162 \mathrm{pb}^{-1}$ data, CDF obtains the following observed (expected) 95\% C.L. upper limits: $\sigma_{s}<13.6(12.1) \mathrm{pb}, \sigma_{t}<10.1(11.2) \mathrm{pb}$ and $\sigma_{s+t}<17.8(13.6) \mathrm{pb}$. The world's best limits are obtained by $\mathrm{D} \emptyset$ from $\sim 370 \mathrm{pb}^{-1}$ of data as a result of their more sophisticated analysis: $\sigma_{s}<5.0(3.3) \mathrm{pb}$ and $\sigma_{t}<4.4(4.3) \mathrm{pb}$. Both collaborations continue to add more data and improve their analyses and more sensitive results are expected soon.

\section{2 $W$ Boson Helicity in Top Quark Decays}

While only single top quark production gives direct access to the magnitude of the $t W b$ interaction, $t \bar{t}$ production can still be used to study its Lorentz structure. This is possible because the $W$ boson polarization in top quark decays depends sensitively on the $t W b$ vertex. Within the SM (V-A interaction), only two $W$ boson helicity configurations, $\lambda_{W}=0,-1$, are allowed. The fraction of longitudinal $\left(\lambda_{W}=0\right)$ and left-handed $\left(\lambda_{W}=-1\right) W$ bosons are completely determined by the values of $m_{t}, M_{W}$ and $m_{b}$ and predicted to be: $F_{0} \simeq 70 \%$ and $F_{-} \simeq 30 \%$, respectively (as a result, $F_{+} \simeq 0 \%$ ). The well-known chiral structure of the $W$ interaction to leptons allows to use lepton kinematic distributions such as the $p_{T}$ in the laboratory frame $\left(p_{T \ell}\right)$ or the cosinus of the lepton decay angle in the $W$ boson rest frame with respect to the $W$ direction $\left(\cos \theta_{\ell}^{*}\right)$ to measure the $W$ helicity fractions. The $p_{T \ell}$ method can be applied to both lepton plus jets and dilepton final states. The $\cos \theta_{\ell}^{*}$ method can only be used in the lepton plus jets final state since explicit top quark reconstruction is required.

Current Run II measurements by CDF and D $\varnothing$ are based on $\sim 160-230 \mathrm{pb}^{-1}$ of data and, due to the still limited statistics, only consider the measurement of one $W$ helicity fraction at a time, fixing the other one to the SM prediction. From the combination of the $\cos \theta_{\ell}^{*}$ (see e.g. Fig. 3(left)) and $p_{T \ell}$ methods, CDF has measured $F_{0}=0.27_{-0.21}^{+0.35}$ (stat. + syst.) whereas DØ has set the limit of $F_{+}<0.25$ at $95 \%$ C.L.. The best measurements in Run I yielded[16] $F_{0}=0.56 \pm 0.31$ (stat. + syst.) (DØ) and $F_{+}<0.18$ at $95 \%$ C.L. (CDF). All measurements, although still limited by statistics, are 
consistent with the SM prediction. The large expected samples in Run II should allow to make more sensitive measurements in the near future.

\section{$\mathbf{8 . 3} \mathbf{B}(\mathbf{t} \rightarrow \mathbf{W b}) / \mathbf{B}(\mathbf{t} \rightarrow \mathbf{W q})$}

Assuming a 3-generation and unitary CKM matrix, $B(t \rightarrow W b)=\Gamma(t \rightarrow W b) / \Gamma_{t} \simeq 1$. An observation of $B(t \rightarrow W b)$ significantly deviating from unity would be a clear indication of new physics such as e.g. a fourth fermion generation or a non-SM top quark decay mode. $\Gamma(t \rightarrow W b)$ can be directly probed in single top quark production, via the cross section measurement. Top quark decays give access to $R \equiv B(t \rightarrow W b) / B(t \rightarrow W q)$, with $q=d, s, b$, which can be expressed as $R=\frac{\left|V_{t b}\right|^{2}}{\left|V_{t d}\right|^{2}+\left|V_{t s}\right|^{2}+\left|V_{t b}\right|^{2}}$, and it's also predicted in the SM to be $R \simeq 1$.

$R$ can be measured by comparing the number of $t \bar{t}$ candidates with 0,1 and $2 b$-tagged jets, since the tagging efficiencies for jets originating from light $(d, s)$ and $b$ quarks are very different. In Run I, CDF measured[17] $R=0.94_{-0.24}^{+0.31}$ (stat. + syst.). In Run II, both CDF and DØ have performed this measurement using data samples of $\sim 160 \mathrm{pb}^{-1}$ and $\sim 230 \mathrm{pb}^{-1}$, respectively. CDF considers events in both the lepton plus jets and dilepton channels and measures[18] $R=$ $1.12_{-0.23}^{+0.27}$ (stat. + syst.), whereas DØ only considers events in the lepton plus jets channel and measures $R=1.03_{-0.17}^{+0.19}$ (stat. + syst.). All measurements are consistent with the SM prediction.

\section{FCNC Couplings of the Top Quark}

Within the SM, neutral-current interactions are flavor-diagonal at tree level. Flavor Changing Neutral Current (FCNC) effects are loop-induced and thus heavily suppressed (e.g. $B(t \rightarrow c g) \simeq$ $10^{-10}, B(t \rightarrow c \gamma / Z) \simeq 10^{-12}$ ), so an observation would be a clear signal of new physics. Indeed, these effects can be significantly enhanced (by factors $\sim 10^{3}-10^{4}$ ) in particular extensions of the SM. Searches for FCNC interactions have been carried out in $p \bar{p}, e^{+} e^{-}$and $e^{ \pm} p$ collisions. At Tevatron, FCNC couplings can manifest themselves both in the form of anomalous single top quark production ( $q g \rightarrow t, q=u, c$ ) or anomalous top quark decays ( $t \rightarrow q V, q=u, c$ and $V=g, \gamma, Z$ ). Only the latter has been experimentally explored so far, via the search for $t \rightarrow q \gamma / Z$ decays.[19] The same $t q \gamma / Z$ interaction would be responsible for anomalous single top quark production in $e^{+} e^{-}\left(e^{+} e^{-} \rightarrow \gamma^{*} / Z \rightarrow t q\right)$ and $e^{ \pm} p(e q \rightarrow e t)$ collisions, and searches have been performed at LEP[20] and HERA,[21] respectively. Fig. 3(right) shows the existing 95\% upper limits on the magnitude of the $t u Z$ and $t u \gamma$ couplings.

Recently, $\mathrm{H} 1$ has reported[21] a $2.2 \sigma$ excess in their search for single top quark production in the leptonic channels. A total of 5 events were observed, compared to $1.31 \pm 0.22$ events expected. No excess was observed in the hadronic channel. The combination of all channels yields a production cross section of $0.29_{-0.14}^{+0.15} \mathrm{pb}$. Interpreted as FCNC-mediated single top quark production, this measurement translates into $\left|\kappa_{t u \gamma}\right|=0.20_{-0.06}^{+0.05}$. Higher statistics measurements at the Tevatron Run II and HERA-II should be able to confirm or exclude this measurement.

\section{Searches for New Particles in Top Quark Production and Decay}

Many models beyond the SM predict new particles preferentially coupled to the top quark: heavy vector gauge bosons (e.g. $q \bar{q} \rightarrow Z^{\prime} \rightarrow t \bar{t}$ in Topcolor), charged scalars (e.g. $t \rightarrow H^{+} b$ in 
generic 2HDM), neutral scalars (e.g. $g g \rightarrow \eta_{T} \rightarrow t \bar{t}$ in Technicolor) or exotic quarks (e.g. $q \bar{q} \rightarrow$ $W^{*} \rightarrow t \overline{b^{\prime}}$ in $E_{6}$ GUT). Because of the large spectrum of theoretical predictions, experimentally it is very important to develop searches as model-independent as possible. These analyses usually look for deviations in kinematic properties (e.g. $t \bar{t}$ invariant mass or top $p_{T}$ spectrum), compare cross section measurements in different decay channels, etc.

In Run II, CDF and DØ are performing model-independent searches for a narrow heavy resonance $X$ decaying to $t \bar{t}$ in the lepton plus $\geq 4$ jets channel. The obtained experimental upper limits on $\sigma_{X} \times B(X \rightarrow t \bar{t})$ vs $M_{X}$ are used to exclude a leptophobic $X$ boson[22] with $M_{X}<700 \mathrm{GeV}$ $(\mathrm{CDF})$ and $M_{X}<680 \mathrm{GeV}(\mathrm{D} \emptyset)$ at $95 \%$ C.L..

Recently, CDF has performed a search for $t \rightarrow H^{+} b$ decays in $t \bar{t}$ events. If $M_{H^{+}}<m_{t}-m_{b}$, $t \rightarrow H^{+} b$ competes with $t \rightarrow W^{+} b$ and results in $B(t \rightarrow W b)<1$. Since $H^{ \pm}$decays are different than $W^{ \pm}$decays, $\sigma_{t \bar{t}}$ measurements in the various channels would be differently affected. By performing a simultaneous fit to the observation in the dilepton, lepton plus tau and lepton plus jets channels, $\mathrm{CDF}$ has determined model-dependent exclusion regions in the $\left(\tan \beta, M_{H}^{ \pm}\right)$plane.[23]

\section{New Physics Contamination in Top Quark Samples}

Top quark events constitute one of the major backgrounds to non-SM processes with similar final state signature. As a result, top quark samples could possibly contain an admixture of exotic processes. A number of model-independent searches have been performed at the Tevatron in Run I and Run II.

A slight excess over prediction in the dilepton channel (in particular in the $e \mu$ final state) was observed in Run I.[24] Furthermore, some of these events had anomalously large lepton $p_{T}$ and $\not_{T}$, which called into question their compatibility with SM $t \bar{t}$ production. In Run II, CDF and D continue to scrutinize the dilepton sample. To date, the event kinematics appears to be consistent with SM $t \bar{t}$ production.[25]. Nevertheless, the flavor anomaly persists: the total number of events observed by both CDF and DØ in the $e \mu(e e+\mu \mu)$ final state is 30(11), whereas the SM prediction is $20 \pm 3(12 \pm 1)$. More data is being analyzed and a definite conclusion on the consistency of the dilepton sample with the SM should be reached soon.

\section{Conclusions}

Till the beginning of the LHC, the Tevatron will remain the world's only top quark factory and a comprehensive program of top quark measurements is well underway. The excellent performances of the accelerator and the $\mathrm{CDF}$ and $\mathrm{D} \emptyset$ detectors open a new era of precision measurements in top quark physics, required to unravel the true nature of the top quark and possibly shed light on the EWSB mechanism. This is a largely unexplored territory, and thus it has the potential to reveal signs of new physics preferentially coupled to the top quark. Most existing measurements appear to be in agreement with the SM, but there are a number of tantalizing (although not statistically significant) anomalies, which should definitely be clarified with the large data samples expected from the Tevatron till the end of 2009. Furthermore, techniques developed at the Tevatron to carry out this rich program of precision top quark physics will be an invaluable experience for the LHC. 


\section{References}

[1] CDF Collaboration, F. Abe et al., Phys. Rev. Lett. 74, 2626 (1995); DØCollaboration, S. Abachi et al., Phys. Rev. Lett. 74, 2632 (1995).

[2] R. Bonciani, S. Catani, M.L. Mangano, and P. Nason, Nucl. Phys. B 529, 424 (1998); N. Kidonakis and R. Vogt, Phys. Rev. D 68, 114014 (2003); M. Cacciari et al., JHEP 404, 68 (2004).

[3] S. Eidelman et al., Phys. Lett. B 592, 1 (2004).

[4] CDF Collaboration, D. Acosta et al., Phys. Rev. D 71, 032001 (2005); DØCollaboration, V.M. Abazov et al., hep-physics/0507191.

[5] CDF Collaboration, T. Affolder et al., Phys. Rev. D 64, 032002 (2001); DØCollaboration, V.M. Abazov et al., Phys. Rev. D 67, 012004 (2003).

[6] CDF Collaboration, D. Acosta et al., Phys. Rev. D 72, 032002 (2005).

[7] CDF Collaboration, A. Abulencia et al., hep-ex/0510048.

[8] DØCollaboration, V.M. Abazov et al., Nature 429, 638 (2004).

[9] CDF Collaboration, A. Abulencia et al., hep-ex/0512070.

[10] CDF Collaboration, DØCollaboration and Tevatron Electroweak Working Group, hep-ex/0404010.

[11] CDF Collaboration, DøCollaboration and Tevatron Electroweak Working Group, hep-ex/0507091.

[12] D. Chang, W.FG. Chang, and E. Ma, Phys. Rev. D 59, 091503 (1999); 61, 037301 (2000).

[13] B.W. Harris et al., Phys. Rev. D 66, 054024 (2002); Z. Sullivan, Phys. Rev. D 70, 114012 (2004).

[14] T. Tait and C.-P. Yuan, Phys. Rev. D 63, 014018 (2001).

[15] CDF Collaboration, D. Acosta et al., Phys. Rev. D 71, 012005 (2005); DØCollaboration, V.M. Abazov et al., Phys. Lett. B 622, 265 (2005).

[16] CDF Collaboration, D. Acosta et al., Phys. Rev. D 71, 031101 (2005); DØCollaboration, V.M. Abazov et al., Phys. Lett. B 617, 1 (2005).

[17] CDF Collaboration, T. Affolder et al., Phys. Rev. Lett. 86, 3233 (2001).

[18] CDF Collaboration, D. Acosta et al., Phys. Rev. Lett. 95, 102003 (2005).

[19] CDF Collaboration, F. Abe et al., Phys. Rev. Lett. 80, 2525 (1998).

[20] Most sensitive result: L3 Collaboration, P. Achard et al., Phys. Lett. B 549, 290 (2002).

[21] ZEUS Collaboration, S. Chekanov et al., Phys. Lett. B 559, 153 (2003); H1 Collaboration, A. Aktas et al., Eur. Phys. J. C 33, 9 (2004).

[22] R.M. Harris, C.T. Hill, and S.J. Parke, hep-ph/9911288.

[23] CDF Collaboration, A. Abulencia et al., hep-ex/0510065.

[24] CDF Collaboration, F. Abe et al., Phys. Rev. Lett. 80, 2779 (1998); DØCollaboration, S. Abachi et al., Phys. Rev. Lett. 79, 1203 (1997).

[25] CDF Collaboration, D. Acosta et al., Phys. Rev. Lett. 95, 022001 (2005). 